Table S4. Differential expression/representation of putative apoptosis pathway genes/proteins.

\begin{tabular}{|c|c|c|c|c|}
\hline \multirow[t]{2}{*}{ Description } & \multirow[t]{2}{*}{$\begin{array}{l}\text { GenBank } \\
\text { (Uniprot) } \\
\text { accession Nos. }\end{array}$} & \multicolumn{3}{|c|}{$\begin{array}{l}\text { Differential gene expression/protein } \\
\text { representation } \\
\text { (Infected/Uninfected Log2-fold; } \mathrm{P}<0.05 \text { ) }\end{array}$} \\
\hline & & Midguts & $\begin{array}{l}\text { Salivary } \\
\text { glands }\end{array}$ & Nymphs \\
\hline \multicolumn{5}{|l|}{ Intrinsic pathway } \\
\hline $\begin{array}{l}\text { Voltage-dependent anion- } \\
\text { selective channel or } \\
\text { mitochondrial porin }\end{array}$ & $\begin{array}{l}\text { ISCW000781 } \\
\text { (B7P5X8) }\end{array}$ & $+0.3 / \mathrm{NS}$ & $-0.5 / \mathrm{NS}$ & $\mathrm{NS} / \mathrm{NS}$ \\
\hline Hexokinase & $\begin{array}{l}\text { ISCW012387 } \\
(\mathrm{B} 7 \mathrm{QB} 25)\end{array}$ & $\mathrm{NS} / \mathrm{NS}$ & $\mathrm{NS} / \mathrm{NS}$ & $-0.6 / \mathrm{NF}$ \\
\hline \multirow{2}{*}{$\begin{array}{l}\text { B-cell CLL/lymphoma } 2 \\
\text { protein Bcl-2 }\end{array}$} & $\begin{array}{l}\text { ISCW019088 } \\
\text { (B7PQ11) }\end{array}$ & $\mathrm{NF} / \mathrm{NF}$ & $\mathrm{NS} / \mathrm{NF}$ & $\mathrm{NS} / \mathrm{NF}$ \\
\hline & $\begin{array}{l}\text { ISCW021516 } \\
\text { (B7Q4U7) }\end{array}$ & $\mathrm{NS} / \mathrm{NF}$ & $\mathrm{NS} / \mathrm{NS}$ & $-3.7 / \mathrm{NF}$ \\
\hline $\begin{array}{l}\text { Bcl-2-associated } \\
\text { transcription factor BTF }\end{array}$ & $\begin{array}{l}\text { ISCW003256 } \\
\text { (B7PCD8) }\end{array}$ & $-2.1 / \mathrm{NF}$ & $\mathrm{NS} / \mathrm{NF}$ & $+2.6 / \mathrm{NF}$ \\
\hline \multirow[t]{3}{*}{$\mathrm{Bcl}-2$ interacting protein } & $\begin{array}{l}\text { ISCW008101 } \\
\text { (B7PTH9) }\end{array}$ & $+1.3 / \mathrm{NS}$ & $\mathrm{NF} / \mathrm{NS}$ & $\mathrm{NF} / \mathrm{NF}$ \\
\hline & $\begin{array}{l}\text { ISCW003136 } \\
\text { (B7PDJ3) }\end{array}$ & $+1.9 / \mathrm{NF}$ & $\mathrm{NF} / \mathrm{NF}$ & $\mathrm{NS} / \mathrm{NF}$ \\
\hline & $\begin{array}{l}\text { ISCW015499 } \\
\text { (B7QP11) }\end{array}$ & $-1.9 / \mathrm{NF}$ & $\mathrm{NS} / \mathrm{NF}$ & $+0.4 / \mathrm{NF}$ \\
\hline \multirow[t]{3}{*}{ Apoptosis inhibitor IAP } & $\begin{array}{l}\text { ISCW010694 } \\
\text { (B7Q7F6) }\end{array}$ & $\mathrm{NS} / \mathrm{NF}$ & $\mathrm{NS} / \mathrm{NF}$ & $-3.7 / \mathrm{NF}$ \\
\hline & $\begin{array}{l}\text { ISCW014060 } \\
\text { (B7QLC6) }\end{array}$ & $\mathrm{NS} / \mathrm{NF}$ & $\mathrm{NS} / \mathrm{NF}$ & $+2.0 / \mathrm{NF}$ \\
\hline & $\begin{array}{l}\text { ISCW002567 } \\
\text { (B7P7P7) }\end{array}$ & NF/NS & $\mathrm{NF} / \mathrm{NS}$ & $\mathrm{NF} / \mathrm{NF}$ \\
\hline Cytochrome c & $\begin{array}{l}\text { ISCW008740 } \\
\text { (B7Q2Y4, } \\
\text { Q4PM75) }\end{array}$ & $+0.8 / \mathrm{NS}$ & $-0.4 / \mathrm{NS}$ & $+0.3 / \mathrm{NF}$ \\
\hline \multirow{2}{*}{ Smac/DIABLO } & $\begin{array}{l}\text { ISCW000014 } \\
\text { (B7P1U9) }\end{array}$ & $-0.9 / \mathrm{NF}$ & $\mathrm{NS} / \mathrm{NF}$ & $+0.7 / \mathrm{NF}$ \\
\hline & $\begin{array}{l}\text { ISCW000886 } \\
\text { (B7P5Y4) }\end{array}$ & $-1.9 / \mathrm{NF}$ & NS/NS & $+3.3 / \mathrm{NF}$ \\
\hline HtrA2/Omi serine protease & $\begin{array}{l}\text { ISCW020167 } \\
\text { (B7PZV8) }\end{array}$ & $\mathrm{NS} / \mathrm{NF}$ & $\mathrm{NS} / \mathrm{NS}$ & $-0.9 / \mathrm{NF}$ \\
\hline \multirow[t]{4}{*}{ Caspases } & $\begin{array}{l}\text { ISCW015329 } \\
\text { (B7QNZ9) }\end{array}$ & $\mathrm{NS} / \mathrm{NS}$ & $\mathrm{NF} / \mathrm{NS}$ & $+0.6 / \mathrm{NF}$ \\
\hline & $\begin{array}{l}\text { ISCW022545 } \\
\text { (B7QAN7) }\end{array}$ & $\mathrm{NS} / \mathrm{NF}$ & $\mathrm{NS} / \mathrm{NF}$ & $-1.6 / \mathrm{NF}$ \\
\hline & $\begin{array}{l}\text { ISCW013172 } \\
\text { (B7QF31) }\end{array}$ & $\mathrm{NS} / \mathrm{NS}$ & $\mathrm{NS} / \mathrm{NS}$ & $-2.5 / \mathrm{NF}$ \\
\hline & $\begin{array}{l}\text { ISCW003039 } \\
\text { (B7PDI2) }\end{array}$ & $-1.7 / \mathrm{NS}$ & $-4.8 /-2.4$ & $+1.2 / \mathrm{NF}$ \\
\hline Caspase-2 & $\begin{array}{l}\text { ISCW014750 } \\
\text { (B7QHN6) }\end{array}$ & NS/NS & $\mathrm{NF} / \mathrm{NS}$ & $-2.9 / \mathrm{NF}$ \\
\hline
\end{tabular}




\begin{tabular}{|c|c|c|c|c|}
\hline \multicolumn{5}{|l|}{ Extrinsic pathway } \\
\hline $\begin{array}{l}\text { Fas apoptotic inhibitory } \\
\text { molecule }\end{array}$ & $\begin{array}{l}\text { ISCW011757 } \\
\text { (B7Q584) }\end{array}$ & $-1.5 / \mathrm{NF}$ & $\mathrm{NS} / \mathrm{NF}$ & $-2.7 / \mathrm{NF}$ \\
\hline \multirow{24}{*}{ Fatty acid synthase (FAS) } & $\begin{array}{l}\text { ISCW000257 } \\
\text { (B7P631) }\end{array}$ & $+4.5 /-1.7$ & $\mathrm{NF} /-1.8$ & $-4.3 / \mathrm{NF}$ \\
\hline & $\begin{array}{l}\text { ISCW009053 } \\
\text { (B7PYN9) }\end{array}$ & $+5.6 / \mathrm{NS}$ & $\mathrm{NS} /-1.7$ & $-3.9 / \mathrm{NS}$ \\
\hline & $\begin{array}{l}\text { ISCW010397 } \\
\text { (B7Q724) }\end{array}$ & $\mathrm{NS} / \mathrm{NF}$ & $\mathrm{NF} /-1.8$ & $-5.3 / \mathrm{NF}$ \\
\hline & $\begin{array}{l}\text { ISCW010398 } \\
\text { (B7Q725) }\end{array}$ & $\mathrm{NS} / \mathrm{NF}$ & $\mathrm{NF} / \mathrm{NF}$ & $-3.8 / \mathrm{NF}$ \\
\hline & $\begin{array}{l}\text { ISCW010399 } \\
\text { (B7Q726) }\end{array}$ & $\mathrm{NS} / \mathrm{NF}$ & $\mathrm{NF} / \mathrm{NF}$ & $-4.6 / \mathrm{NF}$ \\
\hline & $\begin{array}{l}\text { ISCW014349 } \\
\text { (B7QKF5) }\end{array}$ & $\mathrm{NS} / \mathrm{NF}$ & $\mathrm{NF} / \mathrm{NF}$ & $\mathrm{NS} / \mathrm{NF}$ \\
\hline & $\begin{array}{l}\text { ISCW014350 } \\
\text { (B7QKF6) }\end{array}$ & $\mathrm{NS} / \mathrm{NF}$ & $\mathrm{NF} / \mathrm{NF}$ & $\mathrm{NF} / \mathrm{NF}$ \\
\hline & $\begin{array}{l}\text { ISCW001913 } \\
\text { (B7PCH5) }\end{array}$ & $\mathrm{NF} / \mathrm{NF}$ & $\mathrm{NF} / \mathrm{NF}$ & $-3.5 / \mathrm{NF}$ \\
\hline & $\begin{array}{l}\text { ISCW014347 } \\
\text { (B7QKF3) }\end{array}$ & $\mathrm{NF} / \mathrm{NF}$ & $\mathrm{NF} / \mathrm{NF}$ & $-3.3 / \mathrm{NF}$ \\
\hline & $\begin{array}{l}\text { ISCW014535 } \\
\text { (B7QGS8) }\end{array}$ & $\mathrm{NF} / \mathrm{NF}$ & $\mathrm{NF} / \mathrm{NF}$ & $-3.1 / \mathrm{NF}$ \\
\hline & $\begin{array}{l}\text { ISCW018177 } \\
\text { (B7PEX3) }\end{array}$ & $\mathrm{NF} / \mathrm{NF}$ & $\mathrm{NF} / \mathrm{NF}$ & $-2.6 / \mathrm{NF}$ \\
\hline & $\begin{array}{l}\text { ISCW004392 } \\
\text { (B7PK76) }\end{array}$ & $\mathrm{NF} / \mathrm{NS}$ & $\mathrm{NF} / \mathrm{NF}$ & NS/NF \\
\hline & $\begin{array}{l}\text { ISCW001073 } \\
\text { (B7P2U7) }\end{array}$ & $\mathrm{NF} / \mathrm{NF}$ & $\mathrm{NF} / \mathrm{NF}$ & NS/NF \\
\hline & $\begin{array}{l}\text { ISCW014149 } \\
\text { (B7QL04) }\end{array}$ & $\mathrm{NF} / \mathrm{NF}$ & $\mathrm{NF} /-1.8$ & NS/NF \\
\hline & $\begin{array}{l}\text { ISCW018176 } \\
\text { (B7PEX2) }\end{array}$ & $\mathrm{NF} / \mathrm{NF}$ & NF/NF & NS/NF \\
\hline & $\begin{array}{l}\text { ISCW024925 } \\
\text { (B7QJQ3) }\end{array}$ & $\mathrm{NF} / \mathrm{NF}$ & NF/NF & $-4.6 / \mathrm{NF}$ \\
\hline & $\begin{array}{l}\text { ISCW009844 } \\
\text { (B7Q3T1) }\end{array}$ & $\mathrm{NF} / \mathrm{NF}$ & NF/NF & $-5.3 / \mathrm{NF}$ \\
\hline & $\begin{array}{l}\text { ISCW008122 } \\
\text { (B7PVK8) }\end{array}$ & $\mathrm{NF} / \mathrm{NF}$ & NF/NF & $-4.8 / \mathrm{NF}$ \\
\hline & $\begin{array}{l}\text { ISCW014534 } \\
\text { (B7QGS7) }\end{array}$ & $\mathrm{NF} / \mathrm{NF}$ & NF/NF & $-3.4 / \mathrm{NF}$ \\
\hline & $\begin{array}{l}\text { ISCW001566 } \\
\text { (B7P4V0) }\end{array}$ & $\mathrm{NF} / \mathrm{NF}$ & NF/NF & $\mathrm{NS} / \mathrm{NF}$ \\
\hline & $\begin{array}{l}\text { ISCW005148 } \\
\text { (B7PFR3) }\end{array}$ & $\mathrm{NF} / \mathrm{NF}$ & NF/NF & NS/NF \\
\hline & $\begin{array}{l}\text { ISCW010394 } \\
\text { (B7Q6N6) }\end{array}$ & $\mathrm{NF} / \mathrm{NF}$ & NF/NF & NS/NF \\
\hline & $\begin{array}{l}\text { ISCW011309 } \\
\text { (B7Q6F3) }\end{array}$ & $\mathrm{NF} / \mathrm{NF}$ & $\mathrm{NF} / \mathrm{NF}$ & $\mathrm{NS} / \mathrm{NF}$ \\
\hline & $\begin{array}{l}\text { ISCW011310 } \\
\text { (B7Q6F4) }\end{array}$ & $\mathrm{NF} / \mathrm{NS}$ & $\mathrm{NF} / \mathrm{NF}$ & $\mathrm{NS} / \mathrm{NF}$ \\
\hline
\end{tabular}




\begin{tabular}{|c|c|c|c|c|}
\hline Death receptor & $\begin{array}{l}\text { ISCW015148 } \\
\text { (B7QNQ9) } \\
\text { ISCW006321 } \\
\text { (B7PMY2) }\end{array}$ & $\begin{array}{l}\mathrm{NS} / \mathrm{NF} \\
\mathrm{NS} / \mathrm{NF}\end{array}$ & $\begin{array}{l}\text { NS/NF } \\
\text { NS/NF }\end{array}$ & $\begin{array}{l}\mathrm{NS} / \mathrm{NF} \\
-1.8 / \mathrm{NF} \\
\end{array}$ \\
\hline \multicolumn{5}{|c|}{ Perforin/Granzyme pathway } \\
\hline \multirow{7}{*}{ Granzyme } & $\begin{array}{l}\text { ISCW001322 } \\
\text { (B7P540) }\end{array}$ & $\mathrm{NS} / \mathrm{NF}$ & $\mathrm{NF} / \mathrm{NF}$ & $-1.3 / \mathrm{NF}$ \\
\hline & $\begin{array}{l}\text { ISCW013112 } \\
\text { (B7QDI9) }\end{array}$ & $\mathrm{NF} / \mathrm{NF}$ & $\mathrm{NF} / \mathrm{NF}$ & $-2.1 / \mathrm{NF}$ \\
\hline & $\begin{array}{l}\text { ISCW003779 } \\
\text { (B7PHH4) }\end{array}$ & $\mathrm{NF} / \mathrm{NF}$ & $\mathrm{NF} / \mathrm{NF}$ & NF/NF \\
\hline & $\begin{array}{l}\text { ISCW000320 } \\
\text { (B7P0L5) }\end{array}$ & $\mathrm{NF} / \mathrm{NF}$ & $\mathrm{NF} / \mathrm{NF}$ & $-2.8 / \mathrm{NF}$ \\
\hline & $\begin{array}{l}\text { ISCW010999 } \\
\text { (B7Q921) }\end{array}$ & $\mathrm{NF} / \mathrm{NS}$ & $\mathrm{NF} / \mathrm{NF}$ & $\mathrm{NF} / \mathrm{NF}$ \\
\hline & $\begin{array}{l}\text { ISCW011206 } \\
\text { (B7Q613) }\end{array}$ & $\mathrm{NF} / \mathrm{NF}$ & $\mathrm{NF} / \mathrm{NF}$ & $\mathrm{NF} / \mathrm{NF}$ \\
\hline & $\begin{array}{l}\text { ISCW011961 } \\
\text { (B7QB06) }\end{array}$ & $\mathrm{NF} / \mathrm{NF}$ & $\mathrm{NF} / \mathrm{NF}$ & $\mathrm{NF} / \mathrm{NF}$ \\
\hline
\end{tabular}

\section{Execution pathway}

Caspase-3

ISCW001741

(B7P6Y4)

ISCW019519

poly ADP-ribose

polymerase, PARP

(B7PWC4)

ISCW017128

(B7PA88)

ISCW019970

(B7PV22)

Alpha fodrin, spectrin

alpha chain

ISCW000012

Caspase-activated DNase,

CAD

(B7P1U8)

ISCW018943

(B7PNY7)

ISCW017650

Inhibitor of CAD, ICAD

(B7PJN6)

$\begin{array}{ccc}\mathrm{NS} / \mathrm{NF} & \mathrm{NS} / \mathrm{NS} & +1.2 / \mathrm{NF} \\ \mathrm{NS} / \mathrm{NS} & \mathrm{NS} / \mathrm{NS} & -1.2 / \mathrm{NF} \\ -1.3 / \mathrm{NF} & +0.7 / \mathrm{NF} & +1.6 / \mathrm{NF} \\ \mathrm{NS} / \mathrm{NS} & \mathrm{NS} / \mathrm{NS} & -2.9 / \mathrm{NF} \\ +1.0 / \mathrm{NS} & +0.9 / \mathrm{NS} & -1.2 / \mathrm{NS} \\ \mathrm{NS} / \mathrm{NF} & \mathrm{NS} / \mathrm{NF} & \mathrm{NS} / \mathrm{NF} \\ \mathrm{NS} / \mathrm{NF} & +3.1 / \mathrm{NF} & \mathrm{NS} / \mathrm{NF}\end{array}$

\section{Other apoptosis-related genes}

Apoptosis-promoting

RNA-binding protein TIA-

1/TIAR

Programmed cell death protein

Bax-mediated apoptosis inhibitor TEGT/BI-1 Hypothetical protein (mitochondrial ribosomal S23/S29 protein)

\begin{tabular}{|c|c|c|c|}
\hline $\begin{array}{l}\text { ISCW014211 } \\
\text { (B7QI53) }\end{array}$ & $+1.2 / \mathrm{NS}$ & $+1.1 / \mathrm{NS}$ & $+1.4 / \mathrm{NS}$ \\
\hline $\begin{array}{l}\text { ISCW006407 } \\
\text { (B7PMJ5) }\end{array}$ & $-0.7 / \mathrm{NS}$ & $\mathrm{NS} / \mathrm{NS}$ & $+1.3 / \mathrm{NF}$ \\
\hline $\begin{array}{l}\text { ISCW016513 } \\
\text { (B7P6D3) }\end{array}$ & $-0.9 / \mathrm{NF}$ & $\mathrm{NS} / \mathrm{NF}$ & $+0.8 / \mathrm{NF}$ \\
\hline $\begin{array}{l}\text { ISCW018655 } \\
\text { (B7PL49) }\end{array}$ & $-0.7 / \mathrm{NF}$ & $\mathrm{NS} / \mathrm{NF}$ & $-0.7 / \mathrm{NF}$ \\
\hline $\begin{array}{l}\text { ISCW005445 } \\
\text { (B7PNA5) }\end{array}$ & $+1.4 / \mathrm{NF}$ & $\mathrm{NS} / \mathrm{NS}$ & $\mathrm{NS} / \mathrm{NF}$ \\
\hline
\end{tabular}




\begin{tabular}{|c|c|c|c|c|}
\hline Ccar1 & $\begin{array}{l}\text { ISCW023286 } \\
\text { (B7QIS5) }\end{array}$ & $+2.6 / \mathrm{NS}$ & $+1.4 / \mathrm{NF}$ & $-0.8 / \mathrm{NF}$ \\
\hline Apoptosis inducing factor & $\begin{array}{l}\text { ISCW005256 } \\
\text { (B7PLJ8) }\end{array}$ & $+2.6 / \mathrm{NF}$ & NS/NS & $\mathrm{NS} / \mathrm{NF}$ \\
\hline $\begin{array}{l}\text { Apoptosis associated } \\
\text { protein with THAP domain }\end{array}$ & $\begin{array}{l}\text { ISCW021993 } \\
\text { (B7QFZ9) }\end{array}$ & $-1.3 / \mathrm{NF}$ & $+1.2 / \mathrm{NF}$ & $\mathrm{NS} / \mathrm{NF}$ \\
\hline Cellular tumor antigen P53 & $\begin{array}{l}\text { ISCW012889 } \\
\text { (B7QF52) }\end{array}$ & $-1.3 / \mathrm{NF}$ & NS/NF & $+0.8 / \mathrm{NF}$ \\
\hline Anamorsin homolog & $\begin{array}{l}\text { ISCW019144 } \\
\text { (B7PP17) }\end{array}$ & $-2.0 / \mathrm{NS}$ & $-1.0 / \mathrm{NS}$ & $\mathrm{NS} / \mathrm{NF}$ \\
\hline $\begin{array}{l}\text { TP53-regulated inhibitor of } \\
\text { apoptosis }\end{array}$ & $\begin{array}{l}\text { ISCW006194 } \\
\text { (B7PKG1) }\end{array}$ & $-2.3 / \mathrm{NF}$ & $-0.9 / \mathrm{NF}$ & $+1.1 / \mathrm{NF}$ \\
\hline \multirow{2}{*}{ Cell death regulator Aven } & $\begin{array}{l}\text { ISCW018605 } \\
\text { (B7PKZ4) }\end{array}$ & $-2.5 / \mathrm{NF}$ & NS/NF & $+1.7 / \mathrm{NF}$ \\
\hline & $\begin{array}{l}\text { ISCW018605 } \\
\text { (B7PKZ4) }\end{array}$ & $-2.5 / \mathrm{NF}$ & NS/NF & $+1.7 / \mathrm{NF}$ \\
\hline Hypothetical protein & $\begin{array}{l}\text { ISCW011866 } \\
\text { (B7QE72) }\end{array}$ & $-2.9 / \mathrm{NF}$ & $+1.1 / \mathrm{NF}$ & $\mathrm{NS} / \mathrm{NF}$ \\
\hline Defender against cell death & $\begin{array}{l}\text { ISCW024010 } \\
\text { (B7P488, } \\
\text { Q4PM70) }\end{array}$ & $+0.8 / \mathrm{NF}$ & $-1.2 / \mathrm{NS}$ & $-1.5 / \mathrm{NF}$ \\
\hline $\begin{array}{l}\text { NADH:ubiquinone } \\
\text { oxidoreductase, B16.6 }\end{array}$ & $\begin{array}{l}\text { ISCW005224 } \\
\text { (B7PFN5) }\end{array}$ & $+1.0 / \mathrm{NS}$ & NS/NS & $-1.2 / \mathrm{NF}$ \\
\hline \multirow{2}{*}{$\begin{array}{l}\text { Death-associated protein } \\
\text { kinase DAPK }\end{array}$} & $\begin{array}{l}\text { ISCW005225 } \\
\text { (B7PFN6) }\end{array}$ & $+3.4 / \mathrm{NF}$ & NS/NS & $\mathrm{NS} / \mathrm{NF}$ \\
\hline & $\begin{array}{l}\text { ISCW020324 } \\
\text { (B7PZW2) }\end{array}$ & NS/NF & $+2.5 / \mathrm{NF}$ & $+0.6 / \mathrm{NF}$ \\
\hline Ataxin-10 & $\begin{array}{l}\text { ISCW022385 } \\
\text { (B7QGH7) }\end{array}$ & $-1.0 / \mathrm{NF}$ & NS/NS & $-1.7 / \mathrm{NF}$ \\
\hline $\begin{array}{l}\text { Apoptosis antagonizing } \\
\text { transcription factor }\end{array}$ & $\begin{array}{l}\text { ISCW007430 } \\
\text { (B7PW14) }\end{array}$ & $\mathrm{NF} / \mathrm{NF}$ & $\mathrm{NF} / \mathrm{NF}$ & $\mathrm{NF} / \mathrm{NF}$ \\
\hline \multirow{2}{*}{$\begin{array}{l}\text { THAP domain containing, } \\
\text { apoptosis associated } \\
\text { protein } 2\end{array}$} & $\begin{array}{l}\text { ISCW024143 } \\
\text { (B7PCQ7) }\end{array}$ & $\mathrm{NF} / \mathrm{NF}$ & NF/NF & $\mathrm{NF} / \mathrm{NF}$ \\
\hline & $\begin{array}{l}\text { ISCW024918 } \\
\text { (B7QIT8) }\end{array}$ & $\mathrm{NF} / \mathrm{NF}$ & $\mathrm{NF} / \mathrm{NF}$ & $\mathrm{NF} / \mathrm{NF}$ \\
\hline $\begin{array}{l}\text { Apoptosis-promoting } \\
\text { RNA-binding protein TIA- } \\
\text { 1/TIAR }\end{array}$ & $\begin{array}{l}\text { ISCW016601 } \\
\text { (B7PBU8) }\end{array}$ & $\mathrm{NF} / \mathrm{NS}$ & NF/NS & $\mathrm{NF} / \mathrm{NF}$ \\
\hline $\begin{array}{l}\text { Apoptosis stimulating of } \\
\text { P53 }\end{array}$ & $\begin{array}{l}\text { ISCW007014 } \\
\text { (B7PSS5) }\end{array}$ & NF/NF & $\mathrm{NF} / \mathrm{NF}$ & $\mathrm{NF} / \mathrm{NF}$ \\
\hline
\end{tabular}

Janus kinase/signal transducers and activators of transcription (JAK/STAT) pathway

$\begin{array}{lllll}\begin{array}{l}\text { JAK (Hopscotch) tyrosine } \\ \text { protein kinase }\end{array} & \begin{array}{l}\text { ISCW016158 } \\ \text { (B7P4T8) }\end{array} & +2.0 / \mathrm{NF} & \mathrm{NS} / \mathrm{NF} & -2.5 / \mathrm{NF} \\ \text { STAT3 } & \begin{array}{l}\text { ISCW005692 } \\ \text { (B7PKW7) }\end{array} & +1.2 / \mathrm{NF} & \mathrm{NS} / \mathrm{NF} & -1.1 / \mathrm{NF}\end{array}$


JAK receptor protein tyrosine phosphatase (Domeless)

ISCW016699

(B7PAS3)

$+2.1 / \mathrm{NF}$

$\mathrm{NS} / \mathrm{NF}$

$-0.9 / \mathrm{NF}$

PIAS Sumo ligase

ISCW005295

(B7PLL7)

$\mathrm{NS} / \mathrm{NF}$

$\mathrm{NS} / \mathrm{NF}$

$\mathrm{NS} / \mathrm{NF}$

SOCS box SH2 domain- ISCW019435

containing protein

(B7PTW4)

$-2.2 / \mathrm{NF}$

$\mathrm{NS} / \mathrm{NF} \quad-1.0 / \mathrm{NF}$

Abbreviations: +, up-regulated/over-represented in infected ticks; -, downregulated/under-represented in infected ticks; NS, no significant differences between infected and uninfected ticks; NF, transcript/protein not found. 